\title{
The Effect of Aggregate Aspect Ratio and Temperature on the Fracture Toughness of a Low Cement Refractory Concrete
}

\author{
Laura Brum Prata, Walter Libardi*, João Baptista Baldo \\ Departamento de Engenharia de Materiais, UFSCar \\ 13565-905 São Carlos - SP, Brazil
}

Received: September 5, 2001; Revised: August 29, 2003

\begin{abstract}
This work investigated the influence of the aggregate's aspect ratio on the fracture behavior of a low cement aluminum silicate refractory castable treated at two different temperatures $\left(110^{\circ} \mathrm{C}\right.$ and $1000{ }^{\circ} \mathrm{C}$ ). The aggregates were cylindrical pellets with an aspect ratio of 1, 2, 3 and 4, produced by extruding a mixture of clay and calcined alumina fired at $1600{ }^{\circ} \mathrm{C}$ for $4 \mathrm{~h}$ to yield mullite $\left(3 \mathrm{Al}_{2} \mathrm{O}_{3} \cdot 2 \mathrm{SiO}_{2}\right)$. The behavior of the R-Curve and other relevant fracture parameters were evaluated based on the "Two Parameter Fracture Model" in a three-point flexure test of single-edge straight through notched specimens. The two temperature treatments produced different degrees of matrix-aggregate adhesion. The larger aspect ratio aggregates were found to promote toughening only in the dried condition, at $110^{\circ} \mathrm{C}$, while the specimens fired at $1000{ }^{\circ} \mathrm{C}$ for $4 \mathrm{~h}$, regardless of their aggregate aspect ratio, displayed no significant toughening. The best results for fired samples, however, were obtained from specimens containing conventional angular aggregates.
\end{abstract}

Keywords: aspect ratio, refractory concrete, aggregate, toughness, $R$-Curve

\section{Introduction}

Monolithic refractories, due to their attractive cost/benefit ratio and good performance under severe thermo-mechanical applications, have received a great deal of attention in recent years. Their industrial market share today exceeds sixty percent of the world's commercialized refractory products. Among these refractories, low cement (LC) refractory castables occupy a relevant position.

Due to their low $\mathrm{CaO}$ content $(<1,5 \%)$, LC refractory castables display high slag corrosion resistance in addition to thermomechanical properties either similar or superior to those of fired refractory bricks of the same class.

Because of the dynamic evolution of these materials' composite microstructure, the thermal stresses generated may lead to premature or catastrophic failure during the first heat-up and service at high temperatures, particularly in materials exhibiting low toughness and/or nonrising R-Curve behavior.

A refractory castable is composed basically of two phases: a matrix phase containing the cementitious agent
(Calcium Aluminate Cement) plus fine refractory aggregates, and a dispersed phase consisting of coarse and medium refractory aggregates ${ }^{1}$. Since the aggregate is, in principle, the strongest phase, it plays an important role in contributing to the occurrence of toughening mechanisms such as crack deflection ${ }^{2,3}$ and grain bridging ${ }^{4}$. These mechanisms are prone to operate in appropriately tailored structures, particularly the ones in coarse-grained ceramic materials. If a model with a composite-like microstructure is assumed to represent these materials, then it can be inferred that the coarse aggregate may strongly influence the crack propagation resistance. This will depend on several parameters, including aggregate morphology and the characteristics of the aggregate/matrix interface.

The global effect produced by the matrix's fracture toughness and the additional toughening mechanisms developed by aggregates may, under certain circumstances, lead to a synergetic increase in toughness with crack growth. This phenomenon is known as the rising R-Curve behavior.

*e-mail: libardi@power.ufscar.br 
In this work, which also involved normal angular aggregates, a comparative evaluation was made of the influence of coarse cylindrical aggregates with different aspect ratios on the fracture toughness and R-Curve behavior of aluminous low cement refractory castable specimens subjected to two different conditions, i.e., dried at $110^{\circ} \mathrm{C} / 24 \mathrm{~h}$ and fired at $1000{ }^{\circ} \mathrm{C} / 4 \mathrm{~h}$. The mass fraction of aggregates in all the compositions was kept constant at $60 \mathrm{wt} . \%$. In order to determine the R-Curve behavior, the approach used was that of the "Two Parameters Fracture" successfully applied to Portland concrete by Ouyang and Shah ${ }^{5}$. The main feature of the technique is the determination of fracture parameters without the need for measuring crack length during testing. Furthermore, testing involves only one unloading procedure after peak-load, in a simple Load vs. Crack Mouth Opening Displacement (CMOD) three point bend testing.

\section{Materials and Methods}

Cylinder-shaped mullite-rich $\left(3 \mathrm{Al}_{2} \mathrm{O}_{3} \cdot 2 \mathrm{SiO}_{2}\right) 4$ and $2 \mathrm{~mm}$ diameter refractory pellets with lengths ranging from 1 to 4 times the diameter were produced by extruding a mixture of refractory plastic clay plus calcined alumina and firing the pellets for $4 \mathrm{~h}$ at $1600^{\circ} \mathrm{C}$. These pellets were used as the aggregate comprising the coarse fraction of a typical low cement aluminous $\left(65 \mathrm{wt} . \% \mathrm{Al}_{2} \mathrm{O}_{3}\right.$ ) refractory castable mix, in which the coarse aggregate mass fraction was kept constant at $60 \mathrm{wt} . \%$. The calcium aluminate cement $\left(70 \% \mathrm{Al}_{2} \mathrm{O}_{3}\right)$ content was set at $5 \mathrm{wt} . \%$, with the intermediate and fine aggregates comprising $30 \mathrm{wt} . \%$ of the total mass. Based on the specific aspect ratio of the coarse aggregates (from 1 to 4), the compositions were dubbed AR1, AR2, AR3 and AR4. For the sake of comparison, additional specimens of a typical composition of a low cement refractory castable containing regular angular aggregates $(\mathrm{C})$ with a similar chemi$\mathrm{cal} /$ mineralogical composition as that of the cylindrical specimens were prepared. Specimens were also prepared only from the matrix materials constituents (M). Bar-shaped specimens $(4 \times 4 \times 20 \mathrm{~cm})$ were molded under vibration. After curing for $24 \mathrm{~h}$ at room temperature, a set of specimens were dried at $110^{\circ} \mathrm{C} / 24 \mathrm{~h}$, while another set was fired at $1000{ }^{\circ} \mathrm{C}$ for $4 \mathrm{~h}$. The firing temperature of $1000^{\circ} \mathrm{C}$ was chosen in order to obtain a weaker matrix than the aggregate, thus avoiding extensive matrix/aggregate interface reactions. These reactions (sintering) could develop strong adhesive effects and drastically limit the toughening followup. Strong matrix/aggregate adhesion can create undesirable effects in the shear strength of the aggregate/matrix interface, rendering the composite excessively brittle.

\section{Test Procedures}

The specimens were tested by three-point bending, as illustrated in Fig. 1a. A closed-loop testing machine with

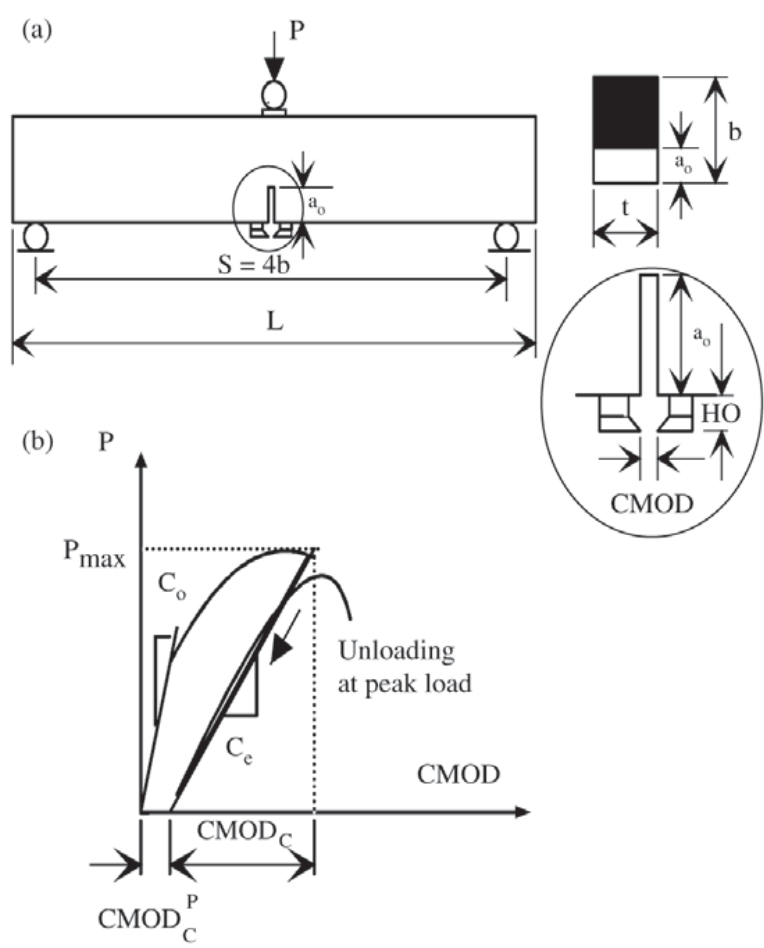

Figure 1. Testing specimen and procedure to determine the values of $K_{I C}^{S}$ and CTOD $_{\mathrm{C}}$.

crack mouth opening displacement (CMOD) feedback was used to produce a stable fracture. The crack mouth opening displacement and the applied load were recorded continuously during the test. A clip gauge was used to measure the CMOD. The loading rate was controlled through a constant CMOD increment rate, enabling the peak load to be reached in about $5 \mathrm{~min}$.

The beam was monotonically loaded up to the maximum load. The applied load was reduced (unloading) manually when it reached approximately $95 \%$ of the maximum load, as depicted in Fig. 1b. When the unloading reached a value of almost zero, the single reloading cycle was started.

\section{Test Results and Calculations}

The modulus of elasticity, $E$, the critical stress intensity factor, $K_{I C}^{S}$, and the critical crack tip opening displacement, $C T O D_{C}$, were determined from the Load $v s$. CMOD curve, using the following correlations ${ }^{6}$ :

$$
E=\frac{6 S a_{0} g\left(\alpha_{0}\right)}{C_{0} b^{2} t}
$$

where $C_{0}$ is the initial compliance calculated from the Load- 
CMOD curve shown in Fig. $1 \mathrm{~b}$ and $g\left(\alpha_{0}\right)$ is given by:

$$
g\left(\alpha_{0}\right)=0.76-2.28 \alpha_{0}+3.87 \alpha_{0}^{2}-2.04 \alpha_{0}^{3}+\frac{0.66}{\left(1-\alpha_{0}\right)^{2}}
$$

where $\alpha_{0}=\left(\alpha_{0}+H O\right) /(b+H O)$ and $S, \alpha_{0}, H O, b$ and $t$ are defined in Fig. 1a.

Based on linear elastic fracture mechanics, an "effective" elastic critical crack length, $\alpha_{c}$, is defined based on the unloading compliance, $C_{e}$, at the peak load. The modulus of elasticity, $E$, can be calculated based on Eq. 1, using the known parameters $C_{0}$ and $\alpha_{0}$. The "effective" elastic critical crack length, $\alpha$, can be evaluated, assuming that the modulus of elasticity does not change ${ }^{6}$ and returning to Eq. 1, in which $C_{0}$ is replaced by $C_{e}$ and $\alpha_{0}$ by $\alpha_{c}$.

The critical stress intensity factor can be calculated from the following equation:

$$
K_{I C}^{S}=\frac{3 P_{c} S \sqrt{\pi a_{c}} f\left(\alpha_{c}\right)}{2 b^{2} t}
$$

where $P_{c}$ is the peak load and the function $f\left(\alpha_{c}\right)$ is given by:

$$
f\left(\alpha_{c}\right)=\frac{1.99-\alpha_{c}\left(1-\alpha_{c}\right)\left(2.15-3.93 \alpha_{c}+2.2 \alpha_{c}^{2}\right)}{\sqrt{\pi}\left(1+2 \alpha_{c}\right)\left(1-\alpha_{c}\right)^{\frac{3}{2}}}
$$

The critical crack tip opening displacement, on the other hand, is evaluated by:

$$
C T O D_{c}=\frac{6 P_{c} S a_{c} g\left(a_{c} / b\right)}{E b^{2} t}\left[\left(1-\beta_{0}\right)^{2}+\left(1.081-1.149 \frac{a_{c}}{b}\right)\left(\beta_{0}-\beta_{0}^{2}\right)\right]^{\frac{1}{2}}
$$

where $b_{0}=\alpha_{d} / \alpha_{c}$ and $g\left(\alpha_{c} / b\right)$ are based on Eq. 2, with $\alpha_{0}$ being replaced by $\alpha_{c} / b$.

Equations 3 and 5 do not take into account the selfweight effect of the beam.

\section{R-Curve}

In the Two Parameter Fracture approach ${ }^{5}$, the R-Curve is defined as an envelope of G-curves with different specimen sizes but with the same notch and specimen geometry. The R-Curve is ${ }^{5}$ formally written as:

$$
\begin{aligned}
& R=\psi\left[1-\left(\frac{d_{2}-\gamma+1}{d_{1}-\gamma+1}\right)\left(\frac{\gamma a_{0}-a_{0}}{a-a_{0}}\right)^{d_{2}-d_{1}}\right]\left(a-a_{0}\right)^{d_{2}} \\
& d_{1,2}=\frac{1}{2}+\frac{\gamma-1}{\gamma} \pm \sqrt{\frac{1}{4}+\frac{\gamma-1}{\gamma}-\left(\frac{\gamma-1}{\gamma}\right)^{2}}
\end{aligned}
$$

Roots $d_{1}$ and $d_{2}$ of Eq. 7 are calculated assigning the positive sign to root $d_{1}$ and the negative sign to root $d_{2}$. The $\gamma$ and $\psi$ parameters can be determined from $K_{I C}^{S}$ and $C T O D_{C}$, as indicated by Eqs. 8 and 9:

$$
\begin{aligned}
& \gamma=\frac{\pi E^{2} f_{1}^{2} C T O D_{c}^{2}}{32 a_{0}\left(K_{I C}^{S}\right)^{2} f_{2}^{2}}+\sqrt{\left[\frac{\pi E^{2} f_{1}^{2} C T O D_{c}^{2}}{32 a_{0}\left(K_{I C}^{S}\right)^{2} f_{2}^{2}}\right]^{2}+1} \\
& \psi=\frac{\left(K_{I C}^{S}\right)^{2}\left(\gamma a_{0}-a_{0}\right)^{-d_{2}}}{E\left(1-\frac{d_{2} \gamma-\gamma+1}{d_{1} \gamma-\gamma+1}\right)}
\end{aligned}
$$

where the single edge notched tensile specimen and the single edge notched three-point bend beam are, respectively, $f_{1}=1.12$ and $f_{2}=1.45$.

It is important to note that the value of $R$ should not exceed the maximum value of $R_{I C}=\left(K_{I C}^{S}\right)^{2} / E$ for $\alpha>\alpha_{c}$. In addition, parameter, which is formally related to and by, expresses the pre-critical stable crack growth and can be considered a toughening index for the material. This means that, if, the material is considered perfectly brittle and devoid of stable crack growth while, if, the material is considered quasi-brittle with stable crack growth.

\section{Results and Discussion}

Figure 2 shows a typical load vs. CMOD curve for unfired specimens. The effect of the aspect ratio of unfired specimens on the fracture and parameters defined above are presented comparatively in Table 1 and Fig. 3. This figure reveals that the specimens containing aggregates with

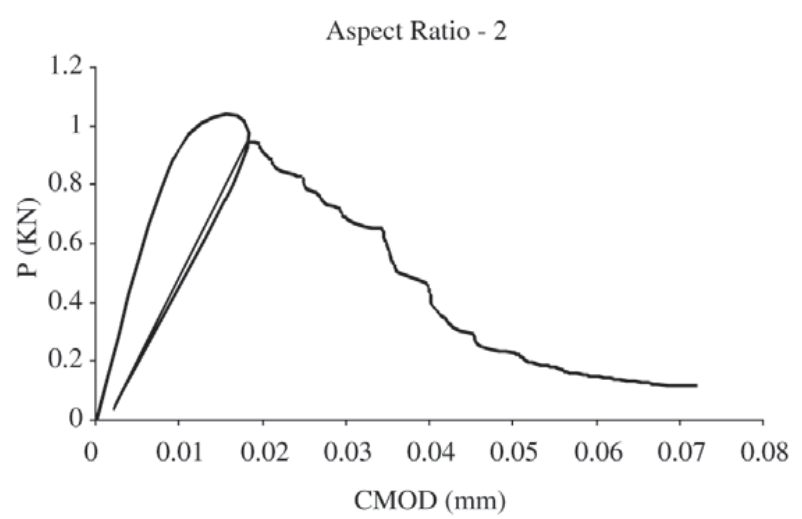

Figure 2. Load vs. CMOD curve for specimen with aggregate of aspect ratio 2 . 
Table 1. Average values of and $R_{I C}$ for each aggregate aspect ratio in unfired specimens.

\begin{tabular}{lcc}
\hline Composition & Aspect Ratio & $\begin{array}{c}\text { Toughening Index } \\
R_{I C}(\mathrm{~N} / \mathrm{mm})\end{array}$ \\
\hline AR1 & 1.17 & 0.0300 \\
AR2 & 1.15 & 0.0292 \\
AR3 & 1.19 & 0.0497 \\
AR4 & 1.26 & 0.0469 \\
C & 1.23 & 0.0180 \\
\hline
\end{tabular}

$\mathbf{C}$ is a conventional low cement aluminous refractory castable containing angular aggregates.

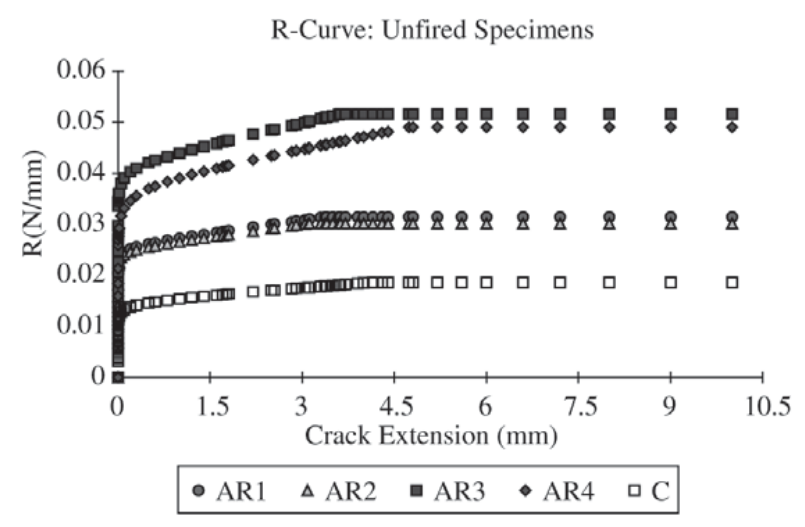

Figure 3. R-Curve of unfired specimens with different compositions and coarse aggregate aspect ratios.

aspect ratios of 1 and 2 display a fracture pattern unlike that of specimens containing aggregates with aspect ratios of 3 and 4. It is clear that fewer fractures occurred in the aggregates in specimens AR1 and AR2 than in those of AR3 and AR4 specimens. The consequences of the different fracture patterns on the fracture parameter values and is evident, i.e., more energy was consumed to fracture specimens AR3 and AR4 than for AR1 and AR2. This indicates an increase in fracture energy for aspect ratios exceeding 2 . In this case (unfired specimens), the composition containing the regular angular aggregates $(\mathrm{C})$ displayed the lowest fracture energy value.

The crack process zone can be estimated from the $\mathrm{R} v s$. Crack Extension plot at the point where the curve reaches a plateau ( $R$ assumes a constant value). Hence, based on Fig. 5 , one can state that the size of the crack process zone of unfired specimens ranged from 3 to $4.0 \mathrm{~mm}$. The size of the crack process appears to be independent of the aggregate aspect ratio.

An analysis of Table 2 and Fig. 4 reveals that firing the samples at $1000{ }^{\circ} \mathrm{C}$ for $4 \mathrm{~h}$ caused the fracture behavior to
Table 2. Average values of and $R_{I C}$ for each aggregate aspect ratio in fired specimens.

\begin{tabular}{lcc}
\hline Composition & Aspect Ratio & $\begin{array}{c}\text { Toughening Index } \\
R_{I C}(\mathrm{~N} / \mathrm{mm})\end{array}$ \\
\hline AR1 & 1.13 & 0.0436 \\
AR2 & 1.12 & 0.0373 \\
AR3 & 1.07 & 0.0364 \\
AR4 & 1.07 & 0.0290 \\
C & 1.14 & 0.0470 \\
M & 1.12 & 0.0399 \\
\hline
\end{tabular}

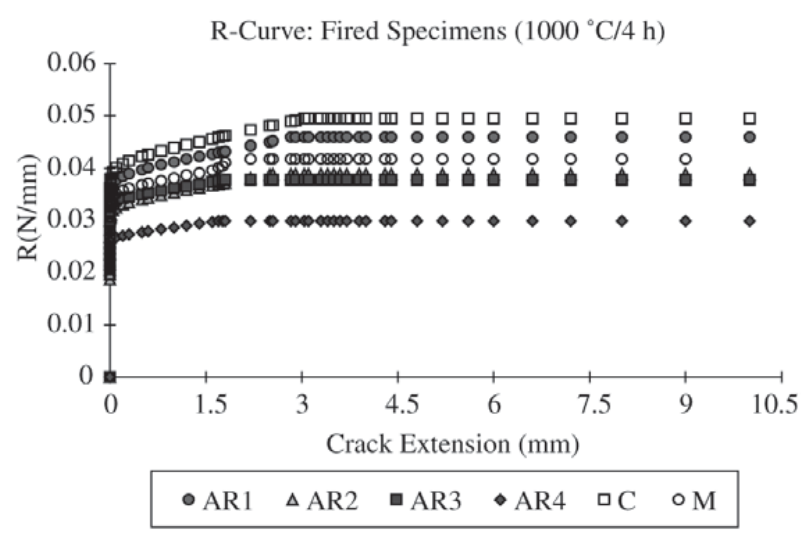

Figure 4. R-Curve for fired $\left(1000{ }^{\circ} \mathrm{C} / 4 \mathrm{~h}\right)$ specimens of different compositions and coarse aggregate aspect ratios.

change drastically in comparison with the unfired specimens. In the $1000{ }^{\circ} \mathrm{C}$ fired condition, the $\alpha$ and $R_{I C}$ values decreased as the aspect ratio increased.

Moreover, two features are visible, the first involving the lower values of $\alpha$ in the fired specimens in contrast with the unfired ones, although the $R_{I C}$ of fired samples increased, with the exception of the unfired AR3 specimens. The highest $R_{I C}$ value was displayed by the low cement castable specimens containing angular aggregates.

The second feature is the shorter length the crack propagated $(2-2.5 \mathrm{~mm})$ to develop its full process zone in the fired specimens, indicating that a relatively low firing temperature sufficed to increase the brittleness of the typical aluminous low cement refractory castable composition investigated.

A clear inversion in the R-Curve behavior was found to occur in the fired condition. In other words, the conventional low cement castable composition containing regular angular aggregates displayed a more strongly increasing R-Curve behavior, followed by the composition containing aggregates with lower aspect ratios. The effect of the in- 
creasing/decreasing R-curve followed the order: $\mathrm{C}>\mathrm{AR} 1>\mathrm{AR} 2 \cong \mathrm{AR} 3>\mathrm{AR} 4$. In this particular situation, the composition containing aggregates of the largest aspect ratio presented an almost flat cleavage-like brittle fracture (Fig. 8).

Interestingly, the global R-Curve behavior of the matrix alone controlled the intermediary situation when compared to the different aspect ratio aggregates containing specimens, as indicated by the data in Table 2 .

From Fig. 5 it is evident that the toughening observed in the unfired specimens for the aggregate aspect ratio exceeding 2 resulted from grain bridging and aggregate pullout. It is well known that rising R-Curve behavior is significantly influenced by bridging and pulling-out processes. This assumption is clearly corroborated by the aggregates protruding from the unfired matrices, resembling (for an aspect

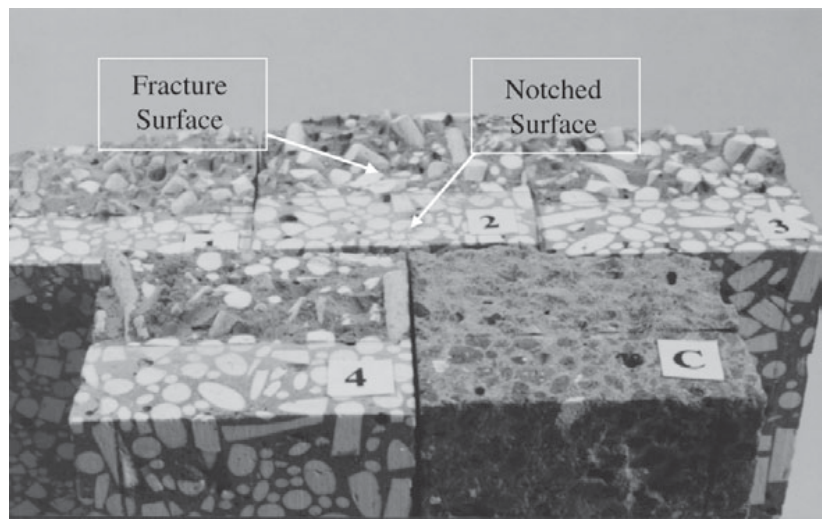

Figure 5. Fracture surface of unfired specimens AR1, AR2, AR3, AR4 and C.

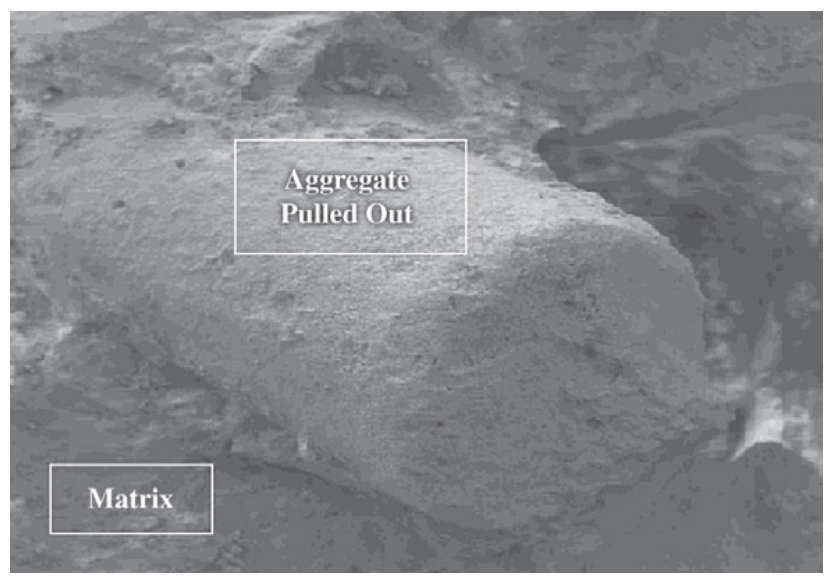

Figure 6. SEM Micrograph of an unfired specimen - Aspect ratio 3. Aggregate protruding from the fracture surface. $(30 \times)$ ratio $\geq 2$ ) the typical fracture surface of a fiber-reinforced composite.

Figures 6 and 7 reveal that the aggregates in the unfired specimens are anchored mostly by mechanical restraint. The aggregate/matrix interface is relatively smooth, with a longitudinal crack located right at the interface, which is free of fracture debris. This indicates pullout without strong disruptive effects on the surrounding matrix.

The fired samples shown in Fig. 8, on the other hand, display no aggregate protrusion, independently of the aggregate aspect ratio. The fracture surface is flat and cleavage-

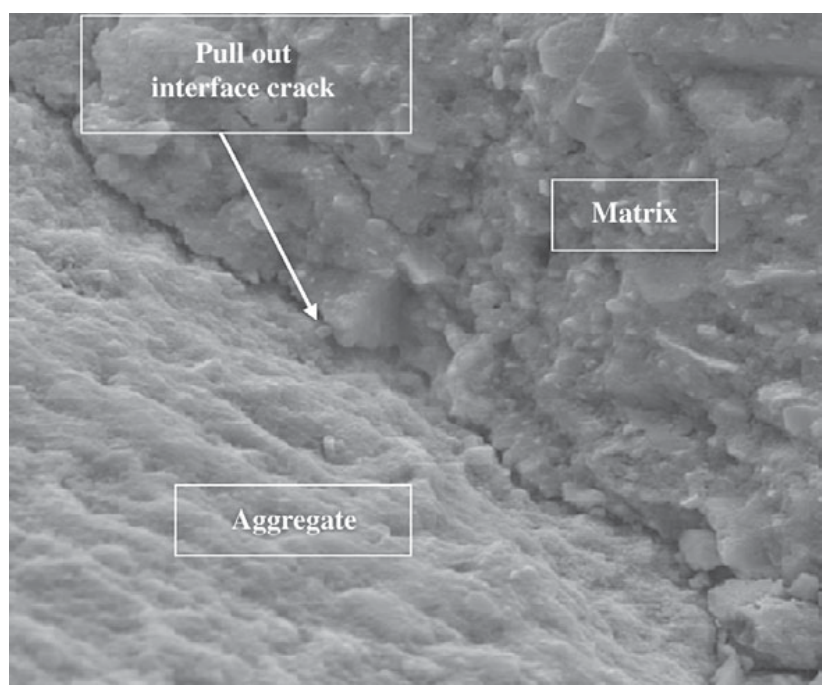

Figure 7. SEM Micrograph of the fracture surface of an unfired specimen - Aspect ratio 3. Longitudinal crack right at the aggregate/matrix interface $(1000 \times)$

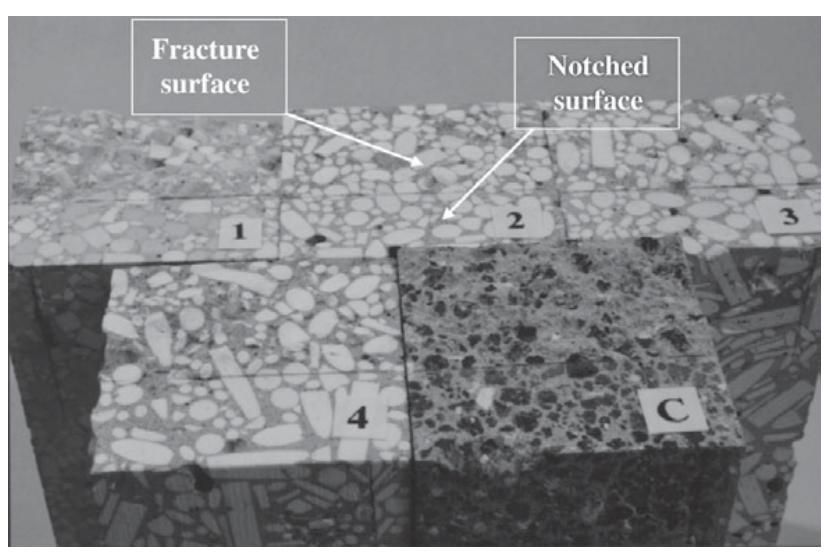

Figure 8. Fracture surface of fired $\left(1000{ }^{\circ} \mathrm{C} / 4 \mathrm{~h}\right)$ specimens AR 1 , AR2, AR3, AR4 and C. 


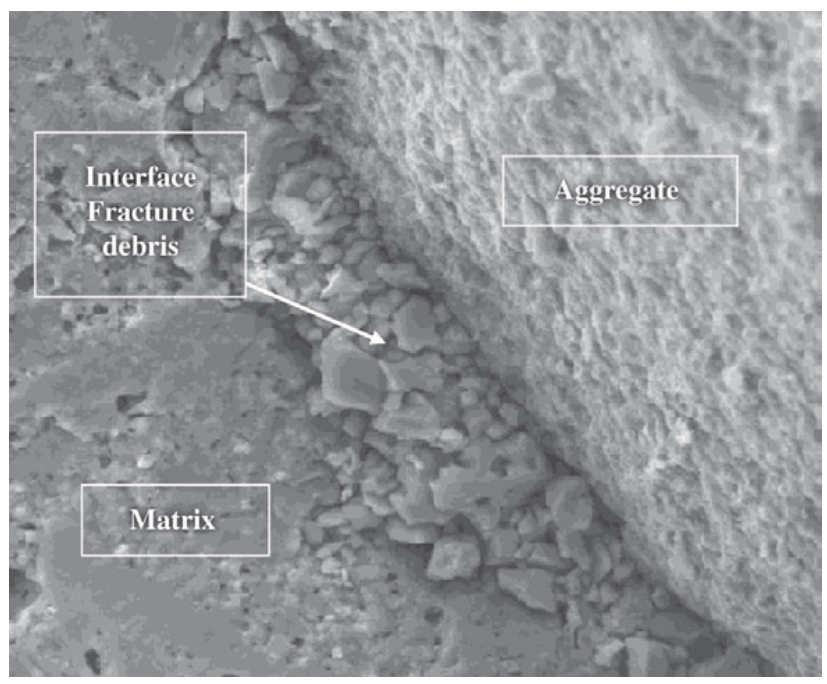

Figure 9. SEM Micrograph of fracture surface of a fired specimen $\left(1000{ }^{\circ} \mathrm{C} / 4 \mathrm{~h}\right)-$ Aspect ratio $3(500 \times)$. Note the fracture debris pilled up at the interface.

like. Figure 9 shows that the aggregate/matrix interface of fired samples is somewhat crumbled, showing a pileup of fracture debris originating from the fracture of strong adhesion points formed due to the incipient sintering between matrix and aggregate.

Aggregate fracture rather than pullout, grain bridging or crack deflection obviously took place in this case. Thus, the incipient toughening observed in the fired samples can be credited only to wake zone effects, such as aggregate wedging and debris shielding by means of friction effects. This situation was more evident in the composition containing regular angular aggregates.

\section{Conclusions}

- An increase of the aspect ratio in unfired specimens of low cement refractory castables promotes increased toughness and R-Curve behavior when the aspect ratio exceeds 2 .
- The increase in the aspect ratio of the coarse aggregate in fired specimens of the low cement refractory castable investigated here promoted no apparent increase in toughness or rising R-Curve behavior.

- The degree of adhesion between the matrix and the aggregate strongly influences the material's brittleness, as shown by the fired specimens.

- Due to possible chemical reactions between the matrix and aggregate, the assumption of a fiber-like composite model for fired refractory castables is inappropriate unless the aggregate/matrix interface adhesion can be controlled.

- Normal angular aggregates promoted improved toughening in the fired condition, indicating that wedging and friction effects on the wake zone were predominant toughening mechanisms in the fired specimens.

\section{Acknowledgements}

The authors would like to thank FAPESP (Process no. 95/0584-2) (State of São Paulo Research Funding Agency) for its financial support of this work, and Prof. Antonio Airton Bortolucci (EESC-USP) and Mr. Benedito O. de Sousa for their help with the testing facilities.

\section{References}

1. Homeny, J.; Bradt, R.C. in Conference on Advanced Materials, Ed.S. Mindess, Westerville,Ohio,Am.Cer.Soc., p. 110, 1991.

2. Faber, K.T.; Evans, A.G. Acta Metall., v. 31, n. 565, 1983.

3. Faber, K.T.; Evans, A.G. Acta Metall., v. 31, n. 577, 1983.

4. Hu, X.Z.; Lutz, E.H.; Swain, M.V. J. Am. Ceram. Soc., v. 74, n. $1828,1991$.

5. Ouyang, C.; Shah, S.P. J. Am. Ceram. Soc., v. 74, n. 2831, 1991.

6. RILEM Comittee on Fracture Mechanics of Concrete Test Methods; - "Determination of Fracture Parameters ( and $C T O D_{C}$ ) of Plain Concrete Using Three-Point Bend Tests" - Mater. Struct., v. 23, p. 457-460, 1990. 